\title{
Attractors for the Stochastic Lattice Selkov Equations with Additive Noises
}

\author{
Hongyan Li \\ College of Management, Shanghai University of Engineering Science, Shanghai, China \\ Email: hongyanlishu@163.com
}

How to cite this paper: Li, H.Y. (2019) Attractors for the Stochastic Lattice Selkov Equations with Additive Noises. Journal of Applied Mathematics and Physics, 7, 1329-1339.

https://doi.org/10.4236/jamp.2019.76090

Received: May 19, 2019

Accepted: June 27, 2019

Published: June 30, 2019

Copyright $\odot 2019$ by author(s) and Scientific Research Publishing Inc. This work is licensed under the Creative Commons Attribution International License (CC BY 4.0).

http://creativecommons.org/licenses/by/4.0/

\begin{abstract}
In this paper, by proving the pullback asymptotic compactness of the stochastic lattice Selkov equations with the cubic nonlinearity, the existence of a random attractor of the stochastic lattice reversible Selkov equations on infinite lattice with additive noises is proved.
\end{abstract}

\section{Keywords}

Random Attractor, Asymptotic Compactness, Selkov System

\section{Introduction}

In this paper, we study that the stochastic lattice Selkov system with the cubic nonlinearity and additive white noises on an infinite lattice is considered in [1] and [2]:

$\left\{\begin{array}{l}\mathrm{d} u_{i}=\left[d_{1}\left(u_{i+1}-2 u_{i}+u_{i-1}\right)-a_{1} u_{i}+b_{1} u_{i}^{2} v_{i}-b_{2} u_{i}^{3}+f_{1 i}\right] \mathrm{d} t+\alpha_{i} \mathrm{~d} w_{i}, \quad i \in \mathbb{Z}, t>0, \\ \mathrm{~d} v_{i}=\left[d_{2}\left(v_{i+1}-2 v_{i}+v_{i-1}\right)-a_{2} v_{i}-b_{1} u_{i}^{2} v_{i}+b_{2} u_{i}^{3}+f_{2 i}\right] \mathrm{d} t+\alpha_{i} \mathrm{~d} w_{i}, \quad i \in \mathbb{Z}, t>0,\end{array}\right.$

with initial conditions

$$
u_{i}(0)=u_{i, 0}, v_{i}(0)=v_{i, 0}, i \in \mathbb{Z},
$$

where $\mathbb{Z}$ denotes the integer set, $u=\left(u_{i}\right)_{i \in \mathbb{Z}} \in \ell^{2}, \quad v=\left(v_{i}\right)_{i \in \mathbb{Z}} \in \ell^{2}$, $d_{1}, d_{2}, a_{1}, a_{2}, b_{1}, b_{2}$ are positive constants, $\alpha=\left(\alpha_{i}\right)_{i \in \mathbb{Z}} \in \ell^{2},\left\{w_{i} \mid i \in \mathbb{Z}\right\}$ is independent Brownian motions.

The reversible Selkov model is derived from a set of the two reversible chemical reactions, which has been studied by [3] [4] and other authors:

$$
P+2 Q \leftrightarrows 3 Q, \quad Q \leftrightarrows Q_{1}
$$

The original Selkov model corresponds to the two irreversible reactions, where the product $Q_{1}$ is an inert product. Let $u_{t}$ and $v_{t}$ are respectively the 
concentrations of the reactants $Q$ and $P$, Equation (1.1) can be regarded as a Selkov system (see [5]) on $\mathbb{R}$ :

$$
\left\{\begin{array}{l}
u_{t}=d_{1} \Delta u-a_{1} u+b_{1} u^{2} v-b_{2} u^{3}+f_{1}+\alpha w_{t}, \\
v_{t}=d_{2} \Delta v-a_{2} v-b_{1} u^{2} v+b_{2} u^{3}+f_{2}+\alpha w_{t} .
\end{array}\right.
$$

For the Equation (1.1), the solution mapping defines a random dynamical system, which is a parametric dynamical system, and pullback absorbing set has been proved, see [1] and [2]. Random attractors are the appropriate objects for describing asymptotic dynamics of such a parametric dynamical system. Therefore, in this paper, we would prove the existence of a random attractor for the stochastic lattice Selkov Equation (1.1).

This paper is organized as follows. In the next section, we recall basic concepts and results related to random attractors. In Section 3, using the transformation of Ornstein-Uhlenbeck process, the stochastic Selkov equation with white noise is transformed into a noiseless determined Selkov equation with random variables as parameters. In Section 4, we prove the pullback asymptotic compactness for the random dynamical system. Then the existence of a random attractor is proved.

\section{Preliminaries}

Firstly, we introduce the relevant definitions of random attractor, which can be taken from [6] [7] [8].

Let $(H, d)$ be a complete separable metric space, $(\Omega, \mathcal{F}, \mathcal{P})$ be a probability space, $\mathbb{R}^{+}=[0, \infty)$.

Definition 2.1. $\left(\Omega, \mathcal{F}, \mathcal{P},\left(\theta_{t}\right)_{t \in \mathbb{R}}\right)$ is called a metric dynamical system if $\theta: \mathbb{R} \times \Omega \rightarrow \Omega$ is $(\mathcal{B}(\mathbb{R}) \times \mathcal{F}, \mathcal{F})$ measurable, $\theta_{0}=I, \theta_{s+t}=\theta_{s} \circ \theta_{t}$ for all $s, t \in \mathbb{R}$, and $\theta_{t} \mathcal{P}=\mathcal{P}$ for all $t \in \mathbb{R}$.

Definition 2.2. A continuous random dynamical system (RDS) on $H$ over a metric dynamical system $\left(\Omega, \mathcal{F}, \mathcal{P},\left(\theta_{t}\right)_{t \in \mathbb{R}}\right)$ is a mapping

$$
\varphi: \mathbb{R}^{+} \times \Omega \times H \rightarrow H, \quad(t, \omega, x) \mapsto \varphi(t, \omega, x),
$$

which is $\left(\mathcal{B}\left(\mathbb{R}^{+}\right) \times \mathcal{F} \times \mathcal{B}(H), \mathcal{B}(H)\right)$-measurable and satisfies, for every $\omega \in \Omega$,

1) $\varphi(0, \omega, \cdot)$ is the identity on $H$;

2) Cocycle property: $\varphi(t+s, \omega, \cdot)=\varphi\left(t, \theta_{s} \omega, \varphi(s, \omega, \cdot)\right)$ for all $t, s \in \mathbb{R}^{+}$;

3) $\varphi(\cdot, \omega, \cdot): \mathbb{R}^{+} \times H \rightarrow H$ is strongly continuous.

Definition 2.3. Suppose $\varphi(t, \omega)$ is a random dynamical system, a random set $\mathcal{A}$ is called a random $\mathcal{D}$ attractor if the following hold:

1) $\mathcal{A}(\omega)$ is a random compact set, i.e., $\omega \rightarrow d(x, \mathcal{A}(\omega))$ is measurable for every $x \in H$ and $\mathcal{A}(\omega)$ is compact for every $\omega \in \Omega$;

2) $\mathcal{A}(\omega)$ is strictly invariant, i.e., for every $\omega \in \Omega$ and all $t \geq 0$ one has $\varphi(t, \omega, \mathcal{A}(\omega))=\mathcal{A}\left(\theta_{t} \omega\right)$;

3) $\mathcal{A}(\omega)$ attracts all sets in $\mathcal{D}$, i.e. for all $B \in \mathcal{D}$ and $\omega \in \Omega$ we have

$$
\lim _{t \rightarrow \infty} d\left(\varphi\left(t, \theta_{-t} \omega, B\left(\theta_{-t} \omega\right)\right), \mathcal{A}(\omega)\right)=0,
$$


where $d(X, Y)=\sup _{x \in X} \inf _{y \in Y}\|x-y\|_{H}$ is the Hausdorff semi-metric (here, $X \subseteq H, Y \subseteq H$ ).

Theorem 2.1 ([9], Proposition 4.1) Let $K(\omega) \in \mathcal{D}$ be an absorbing set for the random dynamical system $\varphi\left(t, \theta_{-t} \omega\right)_{t \geq 0, \omega \in \Omega}$ which is closed and which satisfies for $\omega \in \Omega$ the following asymptotic compactness condition: each sequence $x_{n} \in \varphi\left(t_{n}, \theta_{-t_{n}} \omega, K\left(\theta_{-t_{n}} \omega\right)\right)$ with $t_{n} \rightarrow \infty$ has a convergent subsequence in $H$. Then the random dynamical system $\varphi$ has a unique global random attractor

$$
\mathcal{A}(\omega)=\bigcap_{t \geq t_{K}(\omega) t \geq \tau} \overline{\bigcup_{t \geq \tau} \varphi\left(t, \theta_{-t} \omega, K\left(\theta_{-t} \omega\right)\right)} .
$$

\section{Ornstein-Uhlenbeck Process}

Let $\iota^{2}=\left\{u=\left(u_{i}\right)_{i \in \mathbb{Z}}, u_{i} \in \mathbb{R}: \sum_{i \in \mathbb{Z}}\left|u_{i}\right|^{2}<+\infty\right\}$, with the inner product and norm as follows:

$$
\langle u, v\rangle=\sum_{i \in \mathbb{Z}} u_{i} v_{i},\|u\|^{2}=\langle u, u\rangle, u=\left(u_{i}\right)_{i \in \mathbb{Z}}, v=\left(v_{i}\right)_{i \in \mathbb{Z}} \in t^{2} .
$$

Then $t^{2}=\left(t^{2},\langle\cdot, \cdot\rangle,\|\cdot\|\right)$ is a Hilbert space. Set $E=t^{2} \times t^{2}$ be the product Hilbert space. In view of the cubic term $\pm u^{2} v, \pm u^{3}$, we need $u \in \imath^{6}, v \in \imath^{6}$ to make (1.1) hold in $t^{2}$.

Introducing an Ornstein-Uhlenbeck process (O-U process) (see [10]) in $t^{2}$ on $\left(\Omega, \mathcal{F}, P,\left(\theta_{t}\right)_{t \in \mathbb{R}}\right)$ given by the Wiener process:

$$
y\left(\theta_{t} \omega\right)=-\left(a_{1}+a_{2}\right) \int_{-\infty}^{0} \mathrm{e}^{\left(a_{1}+a_{2}\right) s}\left(\theta_{t} \omega\right)(s) \mathrm{d} s, \quad t \in \mathbb{R}, \omega \in \Omega,
$$

and $y$ solve the Itô equations:

$$
\mathrm{d} y+\left(a_{1}+a_{2}\right) y \mathrm{~d} t=\mathrm{d} w(t), t>0 .
$$

There exists a $\theta_{t}$-invariant set $\Omega^{\prime} \subset \Omega$ of full $P$ measure such that

1) the mappings $s \rightarrow y\left(\theta_{s} \omega\right)$ are continuous for each $\omega \in \Omega$;

2) the random variables $\left\|y\left(\theta_{t} \omega\right)\right\|$ is tempered.

Let

$$
\tilde{u}(t)=u(t)-y\left(\theta_{t} \omega\right), \quad \tilde{v}(t)=v(t)-y\left(\theta_{t} \omega\right) .
$$

From (1.3), we have

$$
\left\{\begin{aligned}
\tilde{u}_{t}= & -d_{1} A\left(\tilde{u}+y\left(\theta_{t} \omega\right)\right)-a_{1} \tilde{u}+a_{2} y\left(\theta_{t} \omega\right)+b_{1}\left(\tilde{u}+y\left(\theta_{t} \omega\right)\right)^{2}\left(\tilde{v}+y\left(\theta_{t} \omega\right)\right) \\
& -b_{2}\left(\tilde{u}+y\left(\theta_{t} \omega\right)\right)^{3}+f_{1} \\
\tilde{v}_{t}= & -d_{2} A\left(\tilde{v}+y\left(\theta_{t} \omega\right)\right)-a_{2} \tilde{v}+a_{1} y\left(\theta_{t} \omega\right)-b_{1}\left(\tilde{u}+y\left(\theta_{t} \omega\right)\right)^{2}\left(\tilde{v}+y\left(\theta_{t} \omega\right)\right) \\
& +b_{2}\left(\tilde{u}+y\left(\theta_{t} \omega\right)\right)^{3}+f_{2}
\end{aligned}\right.
$$

with the initial value condition

$$
\tilde{u}\left(0, \omega, \tilde{u}_{0}\right)=\tilde{u}_{0}(\omega)=u_{0}-y(\omega), \tilde{v}\left(0, \omega, \tilde{v}_{0}\right)=\tilde{v}_{0}(\omega)=v_{0}-y(\omega) .
$$

\section{Pullback Asymptotic Compactness}

From Theorem 2.1, to prove the existence of a random attractor for the random 
dynamical system generated by (1.1), it is necessary to obtain the pullback absorbing property and the pullback asymptotic compactness. The pullback absorbing property has been obtained by [2]. For the pullback asymptotic compactness, we have the following lamma.

Lemma 4.1. Assume the initial functions $\left(u_{0}(\omega), v_{0}(\omega)\right) \in K(\omega)$, where $K(\omega)$ is the absorbing set. Then for every $\varepsilon>0$, there exist $T(\varepsilon, \omega)>0$ and $N(\varepsilon, \omega)>0$ such that the solution $\left(u\left(t, \omega, u_{0}(\omega)\right), v\left(t, \omega, v_{0}(\omega)\right)\right)$ of $(1.1)$ satisfies

$$
\sum_{|i| \geq N(\varepsilon, \omega)}\left[\left\|u\left(t, \theta_{-t} \omega, u_{0}\left(\theta_{-t} \omega\right)\right)\right\|^{2}+\left\|v\left(t, \theta_{-t} \omega, v_{0}\left(\theta_{-t} \omega\right)\right)\right\|^{2}\right]<\varepsilon,
$$

for all $t \geq T(\varepsilon, \omega)>0$.

Proof. We choose a smooth function $\rho$ such that $0 \leq \rho \leq 1$ for all $s \in \mathbb{R}$ and

$$
\rho(s)= \begin{cases}0, & \text { if }|s|<1 \\ 1, & \text { if }|s|>2\end{cases}
$$

and there exists a positive constant $c_{1}$, such that $\left|\rho^{\prime}(s)\right| \leq c_{1}$ for $s \in \mathbb{R}$.

We first consider the random Equation (3.1). Let $r$ be a fixed positive integer which will be specified later. Taking the inner product of the Equation (3.1) with

$$
\begin{aligned}
& \rho\left(\frac{|i|}{r}\right) \tilde{u} \text { and } \rho\left(\frac{|i|}{r}\right) \tilde{v} \text { in } E \text {, respectively, we get } \\
& \frac{1}{2} \frac{\mathrm{d}}{\mathrm{d} t} \sum_{i \in \mathbb{Z}} \rho\left(\frac{|i|}{r}\right)\left|\tilde{u}_{i}\right|^{2} \\
&=-d_{1}\left\langle A \tilde{u}, \rho\left(\frac{|i|}{r}\right) \tilde{u}\right\rangle-d_{1}\left\langle A y\left(\theta_{t} \omega\right), \rho\left(\frac{|i|}{r}\right) \tilde{u}\right\rangle-a_{1} \sum_{i \in \mathbb{Z}} \rho\left(\frac{|i|}{r}\right)\left|\tilde{u}_{i}\right|^{2} \\
&+\left\langle b_{1}\left(\tilde{u}+y\left(\theta_{t} \omega\right)\right)^{2}\left(\tilde{v}+y\left(\theta_{t} \omega\right)\right), \rho\left(\frac{|i|}{r}\right) \tilde{u}\right\rangle-\left\langle b_{2}\left(\tilde{u}+y\left(\theta_{t} \omega\right)\right)^{3}, \rho\left(\frac{|i|}{r}\right) \tilde{u}\right\rangle \\
&+\left\langle f_{1}, \rho\left(\frac{|i|}{r}\right) \tilde{u}\right\rangle+a_{2}\left\langle y\left(\theta_{t} \omega\right), \rho\left(\frac{|i|}{r}\right) \tilde{u}\right\rangle, \\
& \frac{1}{2} \frac{\mathrm{d}}{\mathrm{d} t} \sum_{i \in \mathbb{Z}} \rho\left(\frac{|i|}{r}\right)\left|\tilde{v_{i}}\right|^{2} \\
&=-d_{2}\left\langle\left\langle A \tilde{v}, \rho\left(\frac{|i|}{r}\right) \tilde{v}\right\rangle-d_{2}\left\langle A y\left(\theta_{t} \omega\right), \rho\left(\frac{|i|}{r}\right) \tilde{v}\right\rangle-a_{2} \sum_{i \in \mathbb{Z}} \rho\left(\frac{|i|}{r}\right)|\tilde{v}|^{2}\right. \\
&-\left\langle b_{1}\left(\tilde{u}+y\left(\theta_{t} \omega\right)\right)^{2}\left(\tilde{v}+y\left(\theta_{t} \omega\right)\right), \rho\left(\frac{|i|}{r}\right) \tilde{v}\right\rangle+\left\langle b_{2}\left(\tilde{u}+y\left(\theta_{t} \omega\right)\right)^{3}, \rho\left(\frac{|i|}{r}\right) \tilde{v}\right\rangle \\
&+\left\langle f_{2}, \rho\left(\frac{|i|}{r}\right) \tilde{v}\right\rangle+a_{1}\left\langle y\left(\theta_{t} \omega\right), \rho\left(\frac{|i|}{r}\right) \tilde{v}\right\rangle .
\end{aligned}
$$

By $\left|\rho^{\prime}(s)\right| \leq c_{1}$, we have

$$
\left\langle A \tilde{u}, \rho\left(\frac{|i|}{r}\right) \tilde{u}\right\rangle=\sum_{i \in Z}(B \tilde{u})_{i}\left(B \rho\left(\frac{|i|}{r}\right) \tilde{u}\right)_{i}
$$




$$
\begin{aligned}
& =\sum_{i \in \mathbb{Z}}(B \tilde{u})_{i}\left[\rho\left(\frac{|i+1|}{r}\right) \tilde{u}_{i+1}-\rho\left(\frac{|i|}{r}\right) \tilde{u}_{i}\right] \\
& =\sum_{i \in \mathbb{Z}}\left(\tilde{u}_{i+1}-\tilde{u}_{i}\right)\left[\rho\left(\frac{|i|}{r}\right)\left(\tilde{u}_{i+1}-\tilde{u}_{i}\right)+\left(\rho\left(\frac{|i+1|}{r}\right)-\rho\left(\frac{|i|}{r}\right)\right) \tilde{u}_{i+1}\right] \\
& \geq \sum_{i \in \mathbb{Z}} \rho\left(\frac{|i|}{r}\right)\left|\tilde{u}_{i+1}-\tilde{u}_{i}\right|^{2}-\sum_{i \in \mathbb{Z}} \frac{\rho^{\prime}(\xi)}{r}\left(\tilde{u}_{i+1}-\tilde{u}_{i}\right) \tilde{u}_{i+1} \\
& \geq-\frac{c_{1}}{r} \sum_{i \in \mathbb{Z}}\left(\left|\tilde{u}_{i+1}\right|^{2}+\left|\tilde{u}_{i}\right|\left|\tilde{u}_{i+1}\right|\right) \geq-\frac{2 c_{1}}{r}\|\tilde{u}\|^{2} .
\end{aligned}
$$

And

$$
\left\langle A \tilde{v}, \rho\left(\frac{|i|}{r}\right) \tilde{v}\right\rangle=\sum_{i \in Z}(B \tilde{v})_{i}\left(B \rho\left(\frac{|i|}{r}\right) \tilde{v}\right)_{i} \geq-\frac{2 c_{1}}{r}\|\tilde{v}\|^{2} .
$$

From (4.2), we have

$$
\begin{aligned}
& b_{1}\left\langle\left(\tilde{u}+y\left(\theta_{t} \omega\right)\right)^{2}\left(\tilde{v}+y\left(\theta_{t} \omega\right)\right), \rho\left(\frac{|i|}{r}\right) \tilde{u}\right\rangle-b_{2}\left\langle\left(\tilde{u}+y\left(\theta_{t} \omega\right)\right)^{3}, \rho\left(\frac{i \mid}{r}\right) \tilde{u}\right\rangle \\
& -b_{1}\left\langle\left(\tilde{u}+y\left(\theta_{t} \omega\right)\right)^{2}\left(\tilde{v}+y\left(\theta_{t} \omega\right)\right), \rho\left(\frac{|i|}{r}\right) \tilde{v}\right\rangle+b_{2}\left\langle\left(\tilde{u}+y\left(\theta_{t} \omega\right)\right)^{3}, \rho\left(\frac{|i|}{r}\right) \tilde{v}\right\rangle \\
& =b_{1}\left\langle\left(\tilde{u}+y\left(\theta_{t} \omega\right)\right)^{2}\left(\tilde{v}+y\left(\theta_{t} \omega\right)\right), \rho\left(\frac{|i|}{r}\right)(\tilde{u}-\tilde{v})\right\rangle \\
& -b_{2}\left\langle\left(\tilde{u}+y\left(\theta_{t} \omega\right)\right)^{3}, \rho\left(\frac{|i|}{r}\right)(\tilde{u}-\tilde{v})\right\rangle \\
& \leq \max \left\{b_{1}, b_{2}\right\}\left\langle\left(\tilde{u}+y\left(\theta_{t} \omega\right)\right)^{2}(\tilde{v}-\tilde{u}), \rho\left(\frac{|i|}{r}\right)(\tilde{u}-\tilde{v})\right\rangle \\
& =-\max \left\{b_{1}, b_{2}\right\} \sum_{i \in \mathbb{Z}} \rho\left(\frac{i i \mid}{r}\right)\left(\tilde{u}_{i}+y_{i}\left(\theta_{t} \omega\right)\right)^{2}\left(\tilde{u}_{i}-\tilde{v}_{i}\right)^{2} \leq 0 .
\end{aligned}
$$

Then from (4.2)-(4.5), we find that

$$
\begin{aligned}
& \frac{\mathrm{d}}{\mathrm{d} t} \sum_{i \in \mathbb{Z}} \rho\left(\frac{|i|}{r}\right)\left[\left|\tilde{u}_{i}\right|^{2}+\left|\tilde{v}_{i}\right|^{2}\right]+2 a_{1} \sum_{i \in \mathbb{Z}} \rho\left(\frac{i \mid}{r}\right)\left|\tilde{u}_{i}\right|^{2}+2 a_{2} \sum_{i \in \mathbb{Z}} \rho\left(\frac{i i \mid}{r}\right)\left|\tilde{v}_{i}\right|^{2} \\
\leq & \frac{4 c_{1} d_{1}}{r}\|\tilde{u}\|^{2}+\frac{4 c_{1} d_{2}}{r}\|\tilde{v}\|^{2}+2\left\langle f_{1}, \rho\left(\frac{|i|}{r}\right) \tilde{u}\right\rangle+2\left\langle f_{2}, \rho\left(\frac{|i|}{r}\right) \tilde{v}\right\rangle \\
& -2 d_{1}\left\langle A y\left(\theta_{t} \omega\right), \rho\left(\frac{|i|}{r}\right) \tilde{u}\right\rangle-2 d_{2}\left\langle A y\left(\theta_{t} \omega\right), \rho\left(\frac{|i|}{r}\right) \tilde{v}\right\rangle \\
& +2 a_{2}\left\langle y\left(\theta_{t} \omega\right), \rho\left(\frac{|i|}{r}\right) \tilde{u}\right\rangle+2 a_{1}\left\langle y\left(\theta_{t} \omega\right), \rho\left(\frac{|i|}{r}\right) \tilde{v}\right\rangle .
\end{aligned}
$$

For the third term and forth term in the right-hand side of (4.6), we have

$$
\begin{aligned}
2\left\langle f_{1}, \rho\left(\frac{|i|}{r}\right) \tilde{u}\right\rangle & =\sum_{i \in \mathbb{Z}} \rho\left(\frac{|i|}{r}\right) f_{1 i} \tilde{u}_{i}=\sum_{i|| \geq r} \rho\left(\frac{|i|}{r}\right) f_{1 i} \tilde{u}_{i} \\
& \leq \frac{a_{1}}{3} \sum_{|i| \geq r} \rho\left(\frac{|i|}{r}\right)\left|\tilde{u}_{i}\right|^{2}+\frac{3}{a_{1}} \sum_{i|| \geq r}\left|f_{1 i}\right|^{2},
\end{aligned}
$$




$$
2\left\langle f_{2}, \rho\left(\frac{|i|}{r}\right) \tilde{v}\right\rangle=\sum_{|i| \geq r} \rho\left(\frac{|i|}{r}\right) f_{2 i} \tilde{v}_{i} \leq \frac{a_{2}}{3} \sum_{|i| \geq r} \rho\left(\frac{|i|}{r}\right)\left|\tilde{v}_{i}\right|^{2}+\frac{3}{a_{2}} \sum_{|i| \geq r}\left|f_{2 i}\right|^{2} .
$$

For the fifth term and sixth term in the right-hand side of (4.6), we have

$$
\begin{aligned}
& -2 d_{1}\left\langle A y, \rho\left(\frac{|i|}{r}\right) \tilde{u}\right\rangle=-2 d_{1}\left\langle B y, B\left(\rho\left(\frac{|i|}{r}\right) \tilde{u}\right)\right\rangle \\
& =-2 d_{1} \sum_{i \in \mathbb{Z}}\left(y_{i+1}-y_{i}\right)\left(\rho\left(\frac{|i+1|}{r}\right) \tilde{u}_{i+1}-\rho\left(\frac{|i|}{r}\right) \tilde{u}_{i}\right) \\
& =-2 d_{1} \sum_{|i| \mid r-1} \rho\left(\frac{|i+1|}{r}\right)\left(y_{i+1}-y_{i}\right) \tilde{u}_{i+1}+2 d_{1} \sum_{|i| \geq r} \rho\left(\frac{|i|}{r}\right)\left(y_{i+1}-y_{i}\right) \tilde{u}_{i} \\
& \leq \frac{a_{1}}{3} \sum_{|i| \geq r} \rho\left(\frac{|i|}{r}\right)\left|\tilde{u}_{i}\right|^{2}+C_{1} \sum_{|i| \geq r-1}\left|y_{i}\right|^{2}, \\
& -2 d_{2}\left\langle A y, \rho\left(\frac{|i|}{r}\right) \tilde{v}\right\rangle \leq \frac{a_{2}}{3} \sum_{|i| \geq r} \rho\left(\frac{i i \mid}{r}\right)\left|\tilde{v}_{i}\right|^{2}+C_{2} \sum_{|i| \geq r-1}\left|y_{i}\right|^{2},
\end{aligned}
$$

where $C_{j}, j=1,2$ are positive constants depending only on $d_{1}, d_{2}, a_{1}, a_{2}$. For the last two terms in the right-hand side of (4.6),

$$
\begin{aligned}
& 2 a_{2}\left\langle y\left(\theta_{t} \omega\right), \rho\left(\frac{|i|}{r}\right) \tilde{u}\right\rangle \leq \frac{a_{1}}{3} \sum_{i|| \geq r} \rho\left(\frac{|i|}{r}\right)\left|\tilde{u}_{i}\right|^{2}+\frac{3 a_{2}^{2}}{a_{1}} \sum_{i \in Z} \rho\left(\frac{|i|}{r}\right)\left|y_{i}\left(\theta_{t} \omega\right)\right|^{2}, \\
& 2 a_{1}\left\langle y\left(\theta_{t} \omega\right), \rho\left(\frac{|i|}{r}\right) \tilde{v}\right\rangle \leq \frac{a_{2}}{3} \sum_{i \in Z} \rho\left(\frac{|i|}{r}\right)\left|\tilde{v}_{i}\right|^{2}+\frac{3 a_{1}^{2}}{a_{2}} \sum_{i \in Z} \rho\left(\frac{|i|}{r}\right)\left|y_{i}\left(\theta_{t} \omega\right)\right|^{2} .
\end{aligned}
$$

From (4.6)-(4.9), we have

$$
\begin{aligned}
& \frac{\mathrm{d}}{\mathrm{d} t} \sum_{i \in \mathbb{Z}} \rho\left(\frac{|i|}{r}\right)\left[\left|\tilde{u}_{i}\right|^{2}+\left|\tilde{v}_{i}\right|^{2}\right]+a_{1} \sum_{i \in \mathbb{Z}} \rho\left(\frac{i i \mid}{r}\right)\left|\tilde{u}_{i}\right|^{2}+a_{2} \sum_{i \in \mathbb{Z}} \rho\left(\frac{i \mid}{r}\right)\left|\tilde{v}_{i}\right|^{2} \\
& \leq \frac{4 c_{1} d_{1}}{r}\|\tilde{u}\|^{2}+\frac{4 c_{1} d_{2}}{r}\|\tilde{v}\|^{2}+\frac{3}{a_{1}} \sum_{|i| \geq r}\left|f_{1 i}\right|^{2}+\frac{3}{a_{2}} \sum_{|i| \geq r}\left|f_{2 i}\right|^{2} \\
& \quad+\left(C_{1}+C_{2}\right) \sum_{|i| \geq r-1}\left|y_{i}\left(\theta_{t} \omega\right)\right|^{2}+\left(\frac{3 a_{2}^{2}}{a_{1}}+\frac{3 a_{1}^{2}}{a_{2}}\right) \sum_{|i| \geq r}\left|y_{i}\left(\theta_{t} \omega\right)\right|^{2} .
\end{aligned}
$$

Let $a_{3}=\min \left\{a_{1}, a_{2}\right\}$. By Gronwall's inequality in [11], we have that for $t \geq T_{k}=T_{k}(\omega) \geq 0$,

$$
\begin{aligned}
& \sum_{i \in \mathbb{Z}} \rho\left(\frac{|i|}{r}\right)\left[\left|\tilde{u}_{i}\left(t, \omega, \tilde{u}_{0}(\omega)\right)\right|^{2}+\left|\tilde{v}_{i}\left(t, \omega, \tilde{v}_{0}(\omega)\right)\right|^{2}\right] \\
& \leq \mathrm{e}^{-a_{3}\left(t-T_{k}\right)} \sum_{i \in \mathbb{Z}} \rho\left(\frac{|i|}{r}\right)\left[\left|\tilde{u}_{i}\left(T_{k}, \omega, \tilde{u}_{0}(\omega)\right)\right|^{2}+\left|\tilde{v}_{i}\left(T_{k}, \omega, \tilde{v}_{0}(\omega)\right)\right|^{2}\right] \\
& +\int_{T_{k}}^{t}\left(\left.\frac{4 c_{1} d_{1}}{r}\left\|\left.\tilde{u}\right|^{2}+\frac{4 c_{1} d_{2}}{r}\right\| \tilde{v}\right|^{2}\right) \mathrm{e}^{a_{3}(\tau-t)} \mathrm{d} \tau \\
& +\frac{1}{a_{3}}\left(\frac{3}{a_{1}} \sum_{|i| \geq r}\left|f_{1 i}\right|^{2}+\frac{3}{a_{2}} \sum_{|i| \geq r}\left|f_{2 i}\right|^{2}\right) \\
& +\left(C_{1}+C_{2}\right) \int_{T_{k}}^{t} \mathrm{e}^{a_{3}(\tau-t)} \sum_{|i| \geq r-1}\left|y_{i}\left(\theta_{\tau} \omega\right)\right|^{2} \mathrm{~d} \tau
\end{aligned}
$$




$$
+\left(\frac{3 a_{2}^{2}}{a_{1}}+\frac{3 a_{1}^{2}}{a_{2}}\right) \int_{T_{k}}^{t} \mathrm{e}^{a_{3}(\tau-t)} \sum_{|i| \geq r}\left|y_{i}\left(\theta_{\tau} \omega\right)\right|^{2} \mathrm{~d} \tau .
$$

Replace $\omega$ by $\theta_{-t} \omega$. From (4.11) in [2], with $t$ replaced by $T_{k}$ and $\omega$ by $\theta_{-t} \omega$, it follows that

$$
\begin{aligned}
& \mathrm{e}^{a_{3}\left(t-T_{k}\right)} \sum_{i \in \mathbb{Z}} \rho\left(\frac{|i|}{r}\right)\left[\left.\left|\tilde{u}_{i}\left(T_{k}, \theta_{-t} \omega, \tilde{u}_{0}\left(\theta_{-t} \omega\right)\right)^{2}+\right| \tilde{v}_{i}\left(T_{k}, \theta_{-t} \omega, \tilde{v}_{0}\left(\theta_{-t} \omega\right)\right)\right|^{2}\right] \\
& \leq \mathrm{e}^{a_{3}\left(t-T_{k}\right)}\left(\mathrm{e}^{-a_{3} T_{k}}\left[\left\|\tilde{u}_{0}\left(\theta_{-t} \omega\right)\right\|^{2}+\left\|\tilde{v}_{0}\left(\theta_{-t} \omega\right)\right\|^{2}\right]+\frac{C_{4}}{a_{3}}\left(\left\|f_{1}\right\|^{2}+\left\|f_{2}\right\|^{2}\right)\right. \\
& \left.\quad+C_{4} \int_{0}^{T_{k}} \mathrm{e}^{-a_{3}\left(T_{k}-s\right)}\left(\left\|y\left(\theta_{s-t} \omega\right)\right\|^{2}+\left\|A y\left(\theta_{s-t} \omega\right)\right\|^{2}\right) \mathrm{ds}\right) \\
& \leq \mathrm{e}^{-a_{3} t}\left[\left\|\tilde{u}_{0}\left(\theta_{-t} \omega\right)\right\|^{2}+\left\|\tilde{v}_{0}\left(\theta_{-t} \omega\right)\right\|^{2}\right]+\mathrm{e}^{-a_{3}\left(t-T_{k}\right)} \frac{C_{4}}{a_{3}}\left(\left\|f_{1}\right\|^{2}+\left\|f_{2}\right\|^{2}\right) \\
& \quad+\frac{2}{a_{3}} C_{4} l(\omega) \mathrm{e}^{-\frac{a_{3}}{2}\left(t-T_{k}\right)} .
\end{aligned}
$$

Thus, there exists a $T_{1}(\varepsilon, \omega)>T_{k}(\omega)$ such that if $t>T_{1}(\varepsilon, \omega)$, then

$$
\mathrm{e}^{-a_{3}\left(t-T_{k}\right)} \sum_{i \in \mathbb{Z}} \rho\left(\frac{|i|}{r}\right)\left[\left|\tilde{u}_{i}\left(T_{k}, \theta_{-t} \omega, \tilde{u}_{0}\left(\theta_{-t} \omega\right)\right)\right|^{2}+\left|\tilde{v}_{i}\left(T_{k}, \theta_{-t} \omega, \tilde{v}_{0}\left(\theta_{-t} \omega\right)\right)\right|^{2}\right]<\frac{1}{4} \varepsilon .(
$$

From (4.11), we have

$$
\begin{aligned}
& \int_{T_{k}}^{t}\left(\frac{4 c_{1} d_{1}}{r}\left\|\tilde{u}\left(\tau, \theta_{-t} \omega, \tilde{u}_{0}\left(\theta_{-t} \omega\right)\right)\right\|^{2}+\frac{4 c_{1} d_{2}}{r}\left\|\tilde{v}\left(\tau, \theta_{-t} \omega, \tilde{u}_{0}\left(\theta_{-t} \omega\right)\right)\right\|^{2}\right) \mathrm{e}^{a_{3}(\tau-t)} \mathrm{d} \tau \\
& \leq \frac{4 c_{1} d_{3}}{r} \int_{T_{k}}^{t} \mathrm{e}^{a_{3}(\tau-t)}\left(\mathrm{e}^{-a_{3} \tau}\left[\left\|\tilde{u}_{0}\left(\theta_{-t} \omega\right)\right\|^{2}+\left\|\tilde{v}_{0}\left(\theta_{-t} \omega\right)\right\|^{2}\right]+\frac{C_{4}}{a_{3}}\left(\left\|f_{1}\right\|^{2}+\left\|f_{2}\right\|^{2}\right)\right. \\
& \left.+C_{4} \int_{0}^{\tau} \mathrm{e}^{a_{3}(s-\tau)}\left(\left\|y\left(\theta_{s-t} \omega\right)\right\|^{2}+\left\|A y\left(\theta_{s-t} \omega\right)\right\|^{2}\right) \mathrm{d} s\right) \mathrm{d} \tau \\
& \leq \frac{4 c_{1} d_{3}}{r}\left(\left[\left\|\tilde{u}_{0}\left(\theta_{-t} \omega\right)\right\|^{2}+\left\|\tilde{v}_{0}\left(\theta_{-t} \omega\right)\right\|^{2}\right]\left(t-T_{k}\right) \mathrm{e}^{-a_{3} t}\right. \\
& \left.+\frac{C_{4}}{a_{3}^{2}}\left(\left\|f_{1}\right\|^{2}+\left\|f_{2}\right\|^{2}\right)+\frac{4}{a_{3}} C_{4} l(\omega)\right),
\end{aligned}
$$

where $d_{3}=\max \left\{d_{1}, d_{2}\right\}$. Recall that $\left(\tilde{u}_{0}\left(\theta_{-t} \omega\right), \tilde{v}_{0}\left(\left(\theta_{-t} \omega\right)\right) \in K\left(\theta_{-t} \omega\right)\right.$, which implies that $\left\|\tilde{u}_{0}\left(\theta_{-t} \omega\right)\right\|^{2}+\left\|\tilde{v}_{0}\left(\theta_{-t} \omega\right)\right\|^{2} \leq R^{2}\left(\theta_{-t} \omega\right)$, and $R(\omega)$ is tempered in [2]. Thus there exists $T_{2}(\varepsilon, \omega)>T_{k}(\omega)$ and $N_{1}(\varepsilon, \omega)>0$ such that for $t>T_{2}(\varepsilon, \omega)$ and $r>N_{1}(\varepsilon, \omega)$, we have

$$
\int_{T_{k}}^{t}\left(\frac{4 c_{1} d_{1}}{r}\left\|\tilde{u}\left(\tau, \theta_{-t} \omega, \tilde{u}_{0}\left(\theta_{-t} \omega\right)\right)\right\|^{2}+\frac{4 c_{1} d_{2}}{r}\left\|\tilde{v}\left(\tau, \theta_{-t} \omega, \tilde{u}_{0}\left(\theta_{-t} \omega\right)\right)\right\|^{2}\right) \mathrm{e}^{a_{3}(\tau-t)} \mathrm{d} \tau<\frac{1}{4} \varepsilon .
$$

Since $f_{1}, f_{2} \in t^{2}$, there exists $N_{2}(\varepsilon, \omega)>0$ such that for $r>N_{2}(\varepsilon, \omega)$,

$$
\frac{1}{a_{3}}\left(\frac{3}{a_{1}|i| \geq r}\left|f_{1 i}\right|^{2}+\frac{3}{a_{2}} \sum_{|i| \geq r}\left|f_{2 i}\right|^{2}\right)<\frac{1}{4} \varepsilon \text {. }
$$

Finally, we estimate the last terms on the right-hand side of (4.11). Let $T^{*}>0$ to be determined later. We have for $t>T^{*}+T_{k}$ 


$$
\begin{aligned}
& \left(C_{1}+C_{2}\right) \int_{T_{k}}^{t} \mathrm{e}^{a_{3}(\tau-t)} \sum_{|i| \geq r-1}\left|y_{i}\left(\theta_{\tau-t} \omega\right)\right|^{2} \mathrm{~d} \tau \\
& +\left(\frac{3 a_{2}^{2}}{a_{1}}+\frac{3 a_{1}^{2}}{a_{2}}\right) \int_{T_{k}}^{t} \mathrm{e}^{a_{3}(\tau-t)} \sum_{|i| \geq r}\left|y_{i}\left(\theta_{\tau-t} \omega\right)\right|^{2} \mathrm{~d} \tau \\
& =\int_{T_{k}-t}^{0} \mathrm{e}^{a_{3} s}\left(C_{3} \sum_{|i| \geq r-1}\left|y_{i}\left(\theta_{s} \omega\right)\right|^{2} \mathrm{~d} s+C_{4} \sum_{|i| \geq r}\left|y_{i}\left(\theta_{s} \omega\right)\right|^{2} \mathrm{~d} s\right) \\
& \leq \int_{-T^{*}}^{0} \mathrm{e}^{a_{3} s}\left(C_{3} \sum_{|i| \mid r-1}\left|y_{i}\left(\theta_{s} \omega\right)\right|^{2} \mathrm{~d} s+C_{4} \sum_{|i| \geq r}\left|y_{i}\left(\theta_{s} \omega\right)\right|^{2} \mathrm{~d} s\right) \\
& +\int_{T_{k}-t}^{-T^{*}} \mathrm{e}^{a_{3} s}\left(C_{3}\left\|y\left(\theta_{s} \omega\right)\right\|^{2} \mathrm{~d} s+C_{4}\left\|y\left(\theta_{s} \omega\right)\right\|^{2} \mathrm{~d} s\right) .
\end{aligned}
$$

Using (4.12) in [2], we have

$$
\int_{T_{k}-t}^{-T^{*}} \mathrm{e}^{a_{3} s}\left(C_{3}\left\|y\left(\theta_{s} \omega\right)\right\|^{2}+C_{4}\left\|y\left(\theta_{s} \omega\right)\right\|^{2}\right) \mathrm{d} s \leq \frac{2 C_{5}}{a_{3}} l(\omega) \mathrm{e}^{-\frac{a_{3}}{2} T^{*}} .
$$

Thus, by choosing

$$
T^{*}>\frac{2}{a_{3}} \ln \frac{16 C_{5} l(\omega)}{a_{3} \varepsilon},
$$

we have for $t>T^{*}+T_{k}$,

$$
\int_{T_{k}-t}^{-T^{*}} \mathrm{e}^{a_{3} s}\left(C_{3}\left\|y\left(\theta_{s} \omega\right)\right\|^{2}+C_{4}\left\|y\left(\theta_{s} \omega\right)\right\|^{2}\right) \mathrm{d} s<\frac{\varepsilon}{8} .
$$

For the fixed $T^{*}$, from Lebesgue's theorem, there is $N_{3}(\varepsilon, \omega)$ such that for $r>N_{3}(\varepsilon, \omega)$,

$$
\int_{-T^{*}}^{0} \mathrm{e}^{a_{3} s}\left(C_{3} \sum_{|i| \geq r-1}\left|y_{i}\left(\theta_{s} \omega\right)\right|^{2} \mathrm{~d} s+C_{4} \sum_{|i| \geq r}\left|y_{i}\left(\theta_{s} \omega\right)\right|^{2} \mathrm{~d} s\right)<\frac{\varepsilon}{8} .
$$

Therefore, by letting

$$
\begin{gathered}
T(\varepsilon, \omega)=\max \left\{T_{1}(\varepsilon, \omega), T_{2}(\varepsilon, \omega), T^{*}(\varepsilon, \omega)+T_{k}(\omega)\right\}, \\
\tilde{N}(\varepsilon, \omega)=\max \left\{N_{1}(\varepsilon, \omega), N_{2}(\varepsilon, \omega), N_{3}(\varepsilon, \omega)\right\},
\end{gathered}
$$

for $t>T(\varepsilon, \omega)$ and $N>\tilde{N}(\varepsilon, \omega)$, we have

$$
\begin{aligned}
& \sum_{|i|>2 r}\left[\left|\tilde{u}_{i}\left(t, \theta_{-t} \omega, \tilde{u}_{0}\left(\theta_{-t} \omega\right)\right)\right|^{2}+\left|\tilde{v}_{i}\left(t, \theta_{-t} \omega, \tilde{v}_{0}\left(\theta_{-t} \omega\right)\right)\right|^{2}\right] \\
& \leq \sum_{i \in \mathbb{Z}} \rho\left(\frac{i \mid}{r} \mid\right)\left[\left|\tilde{u}_{i}\left(t, \theta_{-t} \omega, \tilde{u}_{0}\left(\theta_{-t} \omega\right)\right)\right|^{2}+\left|\tilde{v}_{i}\left(t, \theta_{-t} \omega, \tilde{v}_{0}\left(\theta_{-t} \omega\right)\right)\right|^{2}\right] \\
& <\varepsilon,
\end{aligned}
$$

which implies that

$$
\begin{aligned}
& \sum_{|i| \geq N(\varepsilon, \omega)}\left[\left|u_{i}\left(t, \theta_{-t} \omega, u_{0}\left(\theta_{-t} \omega\right)\right)\right|^{2}+\left|v_{i}\left(t, \theta_{-t} \omega, v_{0}\left(\theta_{-t} \omega\right)\right)\right|^{2}\right] \\
= & \sum_{|i| \geq N(\varepsilon, \omega)}\left(\left|\tilde{u}_{i}\left(t, \theta_{-t} \omega, \tilde{u}_{0}\left(\theta_{-t} \omega\right)\right)+y\left(\theta_{-t} \omega\right)\right|^{2}\right. \\
& \left.+\left|\tilde{v}_{i}\left(t, \theta_{-t} \omega, \tilde{v}_{0}\left(\theta_{-t} \omega\right)\right)+y\left(\theta_{-t} \omega\right)\right|^{2}\right)
\end{aligned}
$$




$$
\begin{aligned}
& \leq 2 \sum_{|i| \geq N(\varepsilon, \omega)}\left(\left|\tilde{u}_{i}\left(t, \theta_{-t} \omega, \tilde{u}_{0}\left(\theta_{-t} \omega\right)\right)\right|^{2}+\left|\tilde{v}_{i}\left(t, \theta_{-t} \omega, \tilde{v}_{0}\left(\theta_{-t} \omega\right)\right)\right|^{2}\right) \\
& \quad+6 \sum_{|i| \geq N(\varepsilon, \omega)}\left|y\left(\theta_{-t} \omega\right)\right|^{2} \\
& <8 \varepsilon,
\end{aligned}
$$

provided $N(\varepsilon, \omega)$ is large enough. This completes the proof of the lemma.

We are now ready to show the pullback asymptotic compactness of the random set $K(\omega)$.

Lemma 4.2. For $\omega \in \Omega$, the set $K(\omega)$ is pullback asymptotically compact in the sense of each sequence $\left(u_{n}, v_{n}\right) \in \varphi\left(t_{n}, \theta_{-t_{n}} \omega, K\left(\theta_{-t_{n}} \omega\right)\right)$ with $t_{n} \rightarrow \infty$ having a convergent subsequence in $t^{2} \times l^{2}$.

Proof. We follow the method of [9]. Let $\omega \in \Omega^{\prime}$ for each sequence $\left\{t_{n}\right\}_{n=1}^{\infty}: t_{1}, t_{2}, \cdots, t_{n} \rightarrow \infty$ as $n \rightarrow \infty$, and

$$
\left(u_{n}\left(t_{n}, \theta_{-t_{n}} \omega, x_{n}\right), v_{n}\left(t_{n}, \theta_{-t_{n}} \omega, y_{n}\right)\right) \in \varphi\left(t_{n}, \theta_{-t_{n}} \omega, K\left(\theta_{-t_{n}} \omega\right)\right) ;
$$

this implies that there exists $\left(x_{n}, y_{n}\right) \in K\left(\theta_{-t_{n}} \omega\right)$ such that

$$
\left(u_{n}\left(t_{n}, \theta_{-t_{n}} \omega, x_{n}\right), v_{n}\left(t_{n}, \theta_{-t_{n}} \omega, y_{n}\right)\right)=\varphi\left(t_{n}, \theta_{-t_{n}} \omega,\left(x_{n}, y_{n}\right)\right) .
$$

Since $K(\omega)$ is a bounded absorbing set, for large $n, \varphi\left(t_{n}, \theta_{-t_{n}} \omega,\left(x_{n}, y_{n}\right)\right) \in K(\omega)$; thus there exists $(u, v) \in t^{2} \times t^{2}$, and a subsequence

$$
\begin{aligned}
\left(u_{n}^{\prime}, v_{n}^{\prime}\right)= & \varphi\left(t_{n}, \theta_{-t_{n}} \omega,\left(x_{n}, y_{n}\right)\right) \text { such that } \\
& \left(u_{n}^{\prime}\left(t_{n}, \theta_{-t_{n}} \omega, x_{n}\right), v_{n}^{\prime}\left(t_{n}, \theta_{-t_{n}} \omega, y_{n}\right)\right) \rightarrow(u, v) \text { weak in } \iota^{2} \times l^{2} .
\end{aligned}
$$

Next, we will show that $\left(u_{n}^{\prime}, v_{n}^{\prime}\right)$ is also strongly convergent in the norm $\|\cdot\|$ in $t^{2} \times t^{2}$, i.e., for each $\epsilon>0$ there is $N^{*}(\epsilon, \omega)>0$ such that for $n \geq N^{*}(\epsilon, \omega)$,

$$
\left\|\left(u_{n}^{\prime}\left(t_{n}, \theta_{-t_{n}} \omega, x_{n}\right), v_{n}^{\prime}\left(t_{n}, \theta_{-t_{n}} \omega, y_{n}\right)\right)-(u, v)\right\| \leq \epsilon .
$$

From Lemma 4.1, for any $\epsilon>0$, there exists $N^{*}(\epsilon, \omega)$ and $K_{1}(\epsilon, \omega)$ such that for $n \geq N^{*}(\epsilon, \omega)$,

$$
\sum_{|i| \geq K_{1}(\epsilon, \omega)}\left(\left|u_{n i}^{\prime}\left(t_{n}, \theta_{-t_{n}} \omega, x_{n}\right)\right|^{2}+\left|v_{n i}^{\prime}\left(t_{n}, \theta_{-t_{n}} \omega, y_{n}\right)\right|^{2}\right) \leq \frac{1}{8} \epsilon^{2} .
$$

Since $(u, v) \in t^{2} \times t^{2}$, there exists $K_{2}(\epsilon)>0$ such that

$$
\sum_{|i| \geq K_{2}(\epsilon)}\left(\left|u_{i}\right|^{2}+\left|v_{i}\right|^{2}\right) \leq \frac{1}{8} \epsilon^{2}
$$

Let $K(\epsilon, \omega)=\max \left\{K_{1}(\epsilon, \omega), K_{2}(\epsilon)\right\}$. From the weak convergence (4.24), we have for each $|i| \leq K(\epsilon, \omega)$ as $n \rightarrow \infty$,

$$
\left(u_{n i}^{\prime}\left(t_{n}, \theta_{-t_{n}} \omega, x_{n}\right), v_{n i}^{\prime}\left(t_{n}, \theta_{-t_{n}} \omega, y_{n}\right)\right) \rightarrow\left(u_{i}, v_{i}\right),
$$

which implies that there exists $N_{2}^{*}(\epsilon, \omega)>0$ such that for $n \geq N_{2}^{*}(\epsilon, \omega)$,

$$
\sum_{|i| \leq K(\epsilon, \omega)}\left(\left|u_{n i}^{\prime}\left(t_{n}, \theta_{-t_{n}} \omega, x_{n}\right)-u_{i}\right|^{2}+\left|v_{n i}^{\prime}\left(t_{n}, \theta_{-t_{n}} \omega, y_{n}\right)-v_{i}\right|^{2}\right) \leq \frac{1}{2} \epsilon^{2} .
$$


Combining (4.25)-(4.27), we obtain that for $n \geq N^{*}(\epsilon, \omega)$,

$$
\begin{aligned}
& \left\|u_{n}^{\prime}\left(t_{n}, \theta_{-t_{n}} \omega, x_{n}\right)-u\right\|^{2}+|| v_{n}^{\prime}\left(t_{n}, \theta_{-t_{n}} \omega, y_{n}\right)-v \|^{2} \\
& \leq \sum_{|i| \leq K(\epsilon, \omega)}\left(\left|u_{n i}^{\prime}\left(t_{n}, \theta_{-t_{n}} \omega, x_{n}\right)-u_{i}\right|^{2}+\left|v_{n i}^{\prime}\left(t_{n}, \theta_{-t_{n}} \omega, y_{n}\right)-v_{i}\right|^{2}\right) \\
& \quad+\sum_{|i| \geq K(\epsilon, \omega)}\left(\left|u_{n i}^{\prime}\left(t_{n}, \theta_{-t_{n}} \omega, x_{n}\right)-u_{i}\right|^{2}+\left|v_{n i}^{\prime}\left(t_{n}, \theta_{-t_{n}} \omega, y_{n}\right)-v_{i}\right|^{2}\right) \\
& \leq \frac{1}{2} \epsilon^{2}+2 \sum_{|i| \geq K(\epsilon, \omega)}\left(\left|u_{i}\right|^{2}+\left|v_{i}\right|^{2}\right) \\
& \quad+2 \sum_{|i| \geq K(\epsilon, \omega)}\left(\left|u_{n i}^{\prime}\left(t_{n}, \theta_{-t_{n}} \omega, x_{n}\right)\right|^{2}+\left|v_{n i}^{\prime}\left(t_{n}, \theta_{-t_{n}} \omega, y_{n}\right)\right|^{2}\right) \\
& \leq \frac{1}{2} \epsilon^{2}+\frac{1}{4} \epsilon^{2}+\frac{1}{4} \epsilon^{2}=\epsilon^{2} .
\end{aligned}
$$

Hence, we have completed the proof of Lemma 4.2.

Theorem 4.1. The random dynamical systems $\{\varphi(t, \omega)\}_{t \geq 0, \omega \in \Omega}$ possess a random attractor in $l^{2} \times l^{2}$.

Proof. Note that random dynamical system is pullback asymptotically compact in $E$ by Lemma 4.1 and 4.2. On the other hand, the random dynamical system has a pullback absorbing set by Lemma 4.1 in [2]. Then the existence and uniqueness of a random attractor follow from Theorem 2.1 immediately.

\section{Acknowledgements}

The paper is supported by Shanghai Natural Science Foundation (16ZR1414000), Humanities and Social Sciences Project of the Ministry of Education (16YJCZH043).

\section{Conflicts of Interest}

The author declares no conflicts of interest regarding the publication of this paper.

\section{References}

[1] Li, H. (2019) A Random Dynamical System of the Stochastic Lattice Reversible Selkov Equations. (Submitted)

[2] Li, H. (2019) Pullback Absorbing Set for the Stochastic Lattice Selkov Equations. Asian Research Journal of Mathematics, 14, 1-7. https://doi.org/10.9734/arjom/2019/v14i130116

[3] Chen, H. and Tang, Y. (2019) Proof of Arts-Llibre-Valls's Conjectures for the Higgins-Selkov and the Selkov Systems. Journal of Differential Equations, 11, 7638-7657. https://doi.org/10.1016/j.jde.2018.12.011

[4] Joan, C., Jaume, L. and Vallsb, C. (2018) Dynamics of the Higgins-Selkov and Selkov Systems. Chaos, Solitons and Fractals, 114, 145-150. https://doi.org/10.1016/j.chaos.2018.07.007

[5] Selkov, E. (1968) Self-Oscillations in Glycolysis. I. A Simple Kinetic Model. European Journal of Biochemistry, 4, 79-86. 
https://doi.org/10.1111/j.1432-1033.1968.tb00175.x

[6] Huang, J. (2007) The Random Attractor of Stochastic FitzHugh-Nagumo Equations in an Infinite Lattice with White Noises. Physica D, 233, 83-94.

https://doi.org/10.1016/j.physd.2007.06.008

[7] Li, H. and Tu, J. (2015) Random Attractors for Stochastic Lattice Reversible Gray-Scott Systems with Additive Noise. Electronic Journal of Differential Equations, 2015, 1-25.

[8] Li, H., You, Y. and Tu, J. (2015) Random Attractors and Averaging for Non-Autonomous Stochastic Wave Equations with Nonlinear Damping. Journal of Differential Equations, 258, 148-190. https://doi.org/10.1016/j.jde.2014.09.007

[9] Bates, P.W., Lisei, H. and Lu, K. (2006) Attractors for Stochastic Lattice Dynamical Systems. Stochastics and Dynamics, 6, 1-21.

https://doi.org/10.1142/S0219493706001621

[10] Li, H. (2016) Random Attractor of the Stochastic Lattice Reversible Selkov Equations with Additive Noises. IEEE 13th International Conference on e-Business Engineering, Macau, 4-6 November 2016, 176-181.

https://doi.org/10.1109/ICEBE.2016.038

[11] Témam, R. (1988) Infinite-Dimensional Dynamical Systems in Mechanics and Physics. Applied Mathematical Sciences, Vol. 68, Springer-Verlag, New York. https://doi.org/10.1007/978-1-4684-0313-8 